\title{
Train Detection by Magnetic Field Sensing
}

\author{
Shuqi Zhang, Wing-Kin Lee and Philip Wing-Tat Pong* \\ Room 601, Chow Yei Ching Building, Department of Electrical and Electronic Engineering, \\ The University of Hong Kong, Pokfulam Road, Hong Kong Island, Hong Kong
}

(Received November 5, 2012; accepted February 4, 2013)

Key words: train detection, magnetic field sensing, magnetoresistive sensors, high-speed railway

The recent boom in the development of high-speed railways has stimulated the need for train detection technology to enhance safety and reliability. In this paper, we propose a new technique of train detection through magnetic field sensing by giant magnetoresistive sensors. This technology was studied by the analysis of magnetic field distribution in the high-speed rail system obtained from modeling and simulation. The results verify the feasibility of our technology for the detection of train presence, speed, length, and number of rolling stocks. It can overcome the limitations of track circuits and provide additional measurement capabilities to the signaling system. This detection system is of low cost and minimal maintenance load as well as compacted construction. Thus, it may serve as a new train detection system to help enhance and promote the safety and reliability of high-speed railways.

\section{Introduction}

The recent boom in the development of high-speed railways has motivated enormous research efforts in train speed, ${ }^{(1,2)}$ comfort, ${ }^{(3,4)}$ and safety. ${ }^{(5)}$ Train detection is crucial to the safe operation of high-speed trains. The path of a high-speed train is controlled by a signaling system, which is based on the speed, position, and occupancy status of the trains. ${ }^{(6)}$ The relative position between trains must be determined to avoid train collision and make sure no more than one train can enter a rail section at a time, ensuring the safety, flexibility, and efficiency of train operation. ${ }^{(7)}$

Track circuits are one of the most commonly used train detection devices. ${ }^{(8)}$ One of the major limitations of track circuits is the requirement for insulated rail joints, which weaken the mechanical strength of the rail and have poor compatibility with the highspeed railways operating with welded rails. The adoption of audio frequency signals in a jointless track circuit can alleviate this problem; however, it increases the cost and maintenance load significantly. ${ }^{(9)}$ Moreover, the fuzzy signal at the border and interference of multiple track circuit signals need to be carefully handled.(10) Besides, a track circuit encounters problems at grade crossing where multiple tracks cross, creating permanent shunt paths. A possible solution is to use laser beam detection to compensate

${ }^{*}$ Corresponding author: e-mail: ppong@eee.hku.hk 
for the shortage; however, it would greatly increase the cost of construction. ${ }^{(1)}$ Several factors such as rust, compacted leaf residue, and wet tunnels may cause abnormal operation of the track circuit. The axle counter is also a widely deployed train detection device, which counts the number of wheel pairs passing the detection point at the start of a section. ${ }^{(12)}$ There are several disadvantages of axle counters. In the case when the axle counter loses its memory of the axle number, a manual reset is necessary to restart the system. This introduces human factors as a source of unreliability. Second, axle counters cannot work with a fail-safe principle. Moreover, axle counters have a problem when the wheels stop right at the counters. It may also regard the electromagnetic braking devices on high-speed trains as axles by mistake. ${ }^{(13)}$ Some researchers suggest a combined use of track circuit and axle counter; however, it does not help address the disadvantages mentioned above. ${ }^{(14)}$ There are other types of detection methods. Sense coils, inductive loops, and trip switches can only detect trains in motion, and they cannot detect stationary objects. ${ }^{(15)}$ The EVA system ${ }^{(15)}$ applies infrared beam sensors for stationary train detection, which results in significant expenses. Other detection systems include vibration detection, ${ }^{(16-18)}$ passive infrared and ultrasonic detection, video imaging, sound detection of locomotive horn, communication-based train detection with onboard Global Positioning System (GPS) and wayside radio links. ${ }^{(15)}$ However, these methods either require expensive equipment and large maintenance load or they are susceptible to interference from various factors such as light condition and environmental temperature. Thus, a new detection technique is needed for high-speed railways, which require a higher safety standard and a more stringent signaling.

In this paper, a new train detection technique for high-speed railways by magnetic field sensing with giant magnetoresistive (GMR) sensors is proposed. Magnetic field sensing has been applied in vehicle detection and car speed measurement. ${ }^{(19)}$ Since railway trains contain ferromagnetic materials, it is possible to develop a similar method for train detection. The new technique eliminates the need for insulated rail joints. It makes use of the magnetic field generated from the overhead contact systems of highspeed rails and thus it is compatible with all high-speed railway systems. Moreover, this technique enables continuous detection at grade crossing, and it is applicable to both moving and stationary trains. It offers additional features: it can measure the number of rolling stocks, train speed, and length, which may enable us to obtain the train identity and statistics. GMR sensors offer the best performance in terms of cost, size, and energy consumption among various magnetometers such as anisotropic magnetoresistance (AMR) sensors, fluxgate, superconducting quantum interference devices (SQUIDs), and electron-spin-resonance magnetometers. ${ }^{(20)}$ These advantages are beneficial for largescale application and long-term usage as required for train detection in railways.

\section{Modeling and Simulation}

The modeling and simulation were carried out by the finite element method in ANSYS Maxwell. The entire model is divided into two parts: the electrical circuit for power supply and the train. The circuit consists of overhead contact lines for power supply, which include a catenary wire and a contact wire, and the return circuit for returning current including one negative feeder, one return conductor, two rails, 
and an earth-buried conductor under the ground between the rails, as shown in Fig. 1. The current values, current types, and conductor materials required for modeling and simulation of the electrical circuit are shown in Table 1. The cross sections and coordinates for conductors and wires are illustrated in Table 2. The train model adopted in our simulation is the Deutsche-Bahn ICE 3 high-speed train shown in Fig. 2. The model of the train consists of two heads and six rolling stocks as shown in Fig. 3. The material is stainless steel. The entire model is placed along the $y$ axis [Fig. 4(a)]. The length of all the conductors including two rails is $400 \mathrm{~m}$. The length of the train is 196 $\mathrm{m}$. Each head and rolling stock are 7.8 and $23.8 \mathrm{~m}$, respectively. There is a space of 0.6 $\mathrm{m}$ between neighboring rolling stocks. The whole railway model including two parallel rails is shown in Fig. 4(b). Therefore, the magnetic field from inside the train can be neglected. The detailed settings for the simulation including solution type, number of adaptive passes, percent error, and refinement per pass are listed in Table 3. Simulation was carried out and it was found that the magnetic field sources (e.g., $1000 \mathrm{~A}, 50 \mathrm{~Hz}$ current) inside the train are shielded by the metallic frame of a rolling stock and it cannot penetrate outside the train. Therefore, the magnetic field from inside the train can be neglected.

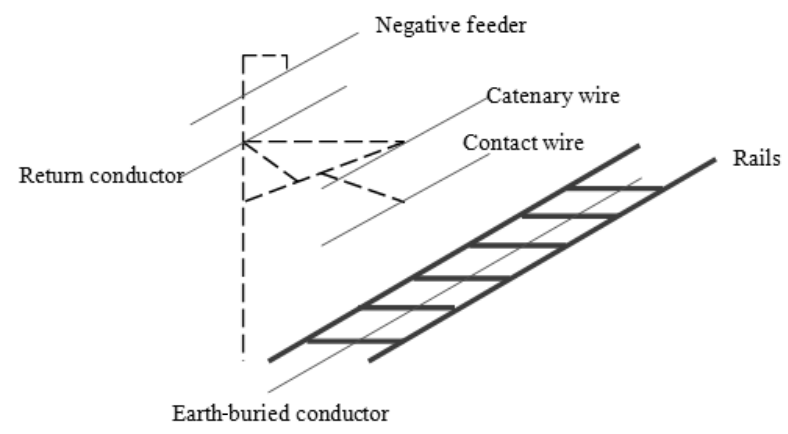

Fig. 1. Electrical circuit model for railway power supply. The dotted lines represent the frame that supports all the wires.

Table 1

Electrical and material details of modeling for overhead contact system. The "+" and "-" signs in front of the current value only indicate the two opposite directions for the current in the close loop circuit. All currents are $50 \mathrm{~Hz} \mathrm{AC}$ in phase.

\begin{tabular}{lccc}
\hline Conductor name & Current value [A] & Current type & Material \\
\hline Catenary wire & +415 & Stranded & Copper \\
Contact wire & +735 & Stranded & Copper \\
Negative feeder & -603 & Stranded & Copper \\
Return conductor & -210 & Stranded & Copper \\
Earth-buried conductor & -74 & Stranded & Copper \\
Right rail & -181.5 & Eddy & Stainless steel \\
Left rail & -181.5 & Eddy & Stainless steel \\
\hline
\end{tabular}


Table 2

Cross sections and coordinates for modeling of overhead contact system.

\begin{tabular}{lccc}
\hline Conductor name & Cross section & Radius $[\mathrm{m}]$ & Coordinates $(x, z)[\mathrm{m}]$ \\
\hline Catenary wire & - & 0.00707 & $(2,9.5)$ \\
Contact wire & - & 0.006 & $(0,5.4)$ \\
Negative feeder & - & 0.006 & $(0,7.2)$ \\
Return conductor & - & 0.006 & $(3,5.4)$ \\
Earth-buried conductor & - & 0.006 & $(0,-0.2)$ \\
Left rail & - & & $(0.7875,0)$ \\
Right rail & - & & $(-0.7875,0)$ \\
\end{tabular}

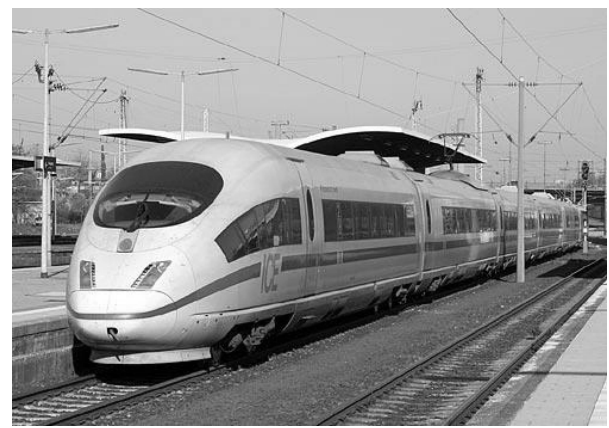

(a)

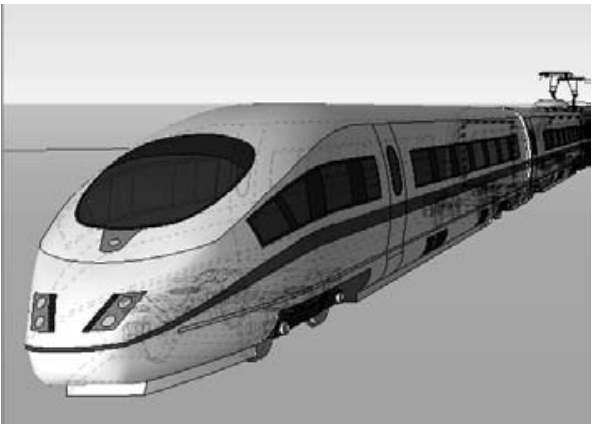

(b)

Fig. 2. (a) Deutsche-Bahn ICE 3 high-speed train and (b) structural model of Deutsche-Bahn ICE 3 from Google sketchup.

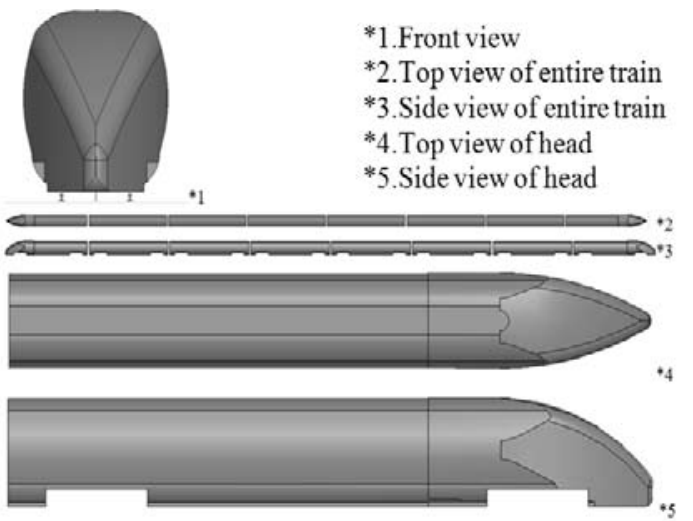

Fig. 3. Train model in different views. 


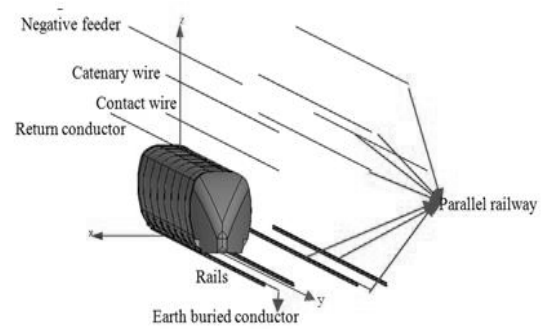

(a)

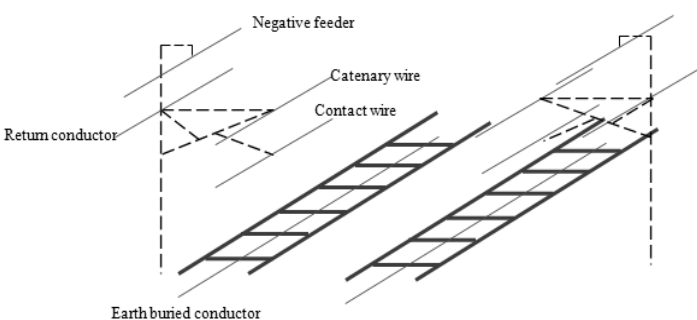

(b)

Fig. 4. Overall 3D view of complete model: (a) established model in Maxwell and (b) illustration of railway structure including two parallel rails.

Table 3

Settings and parameters for simulation.

\begin{tabular}{llc}
\hline Solution type & & Eddy current \\
\hline Model & & $3 \mathrm{D}$ \\
\hline Analysis setup & Passes & 8 \\
& Percent error & 1 \\
& Refinement per pass & 30 \\
& Minimum number of passes & 2 \\
& Minimum converged passes & 1 \\
& Adaptive frequency & $50 \mathrm{~Hz}$ \\
\hline
\end{tabular}

\section{Simulation Results and Discussion}

\subsection{Effect of train presence on magnetic field distribution}

The simulation result in Fig. 5 shows the magnetic field distribution on the $x z$ plane. The magnetic field is strongest around the wires and conductors, and it decays as it is farther away from these sources. By comparing with the situation without a train (the right half of the simulation), it can be observed that the magnetic field distribution becomes dense and strong above the train, while sparse and weak at the sides of the train. This leads to the phenomenon that the presence of the train warps the magnetic field generated from the wires and conductors - the train presence strengthens the field above the train and weakens the field at the sides of the train. Figure 6 shows the effect of train presence on the magnetic field distribution in the top view. The plane selected is right above the train in the $x y$ plane. The outline of the train is overlapped onto the simulation result for better observation. It can be seen that in the area above the train, the magnetic field becomes stronger than in the regions nearby. At the side of the train, the magnetic field is weakened. From the simulation results in both Figs. 5 and 6, the presence of a train warps the magnetic field generated from the wires and conductors. The effect of the warping above the train is opposite to that at the sides of the train. The magnetic field becomes stronger above the train whereas the magnetic field is weakened at the sides. 


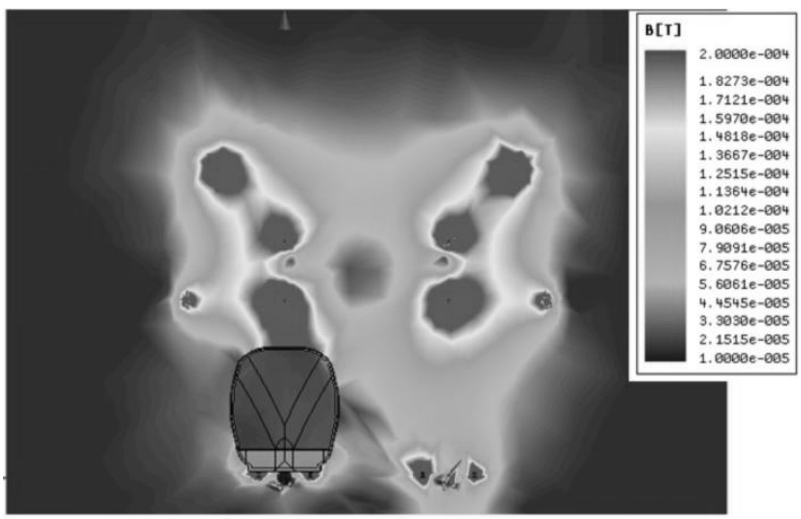

Fig. 5. Magnetic field distribution on $x z$ plane $(y=0)$. The right half shows the distribution of magnetic field without a train. The comparison between the left and right half shows that the magnetic field above the train is strengthened, while the fields at the two sides of the train are weakened.

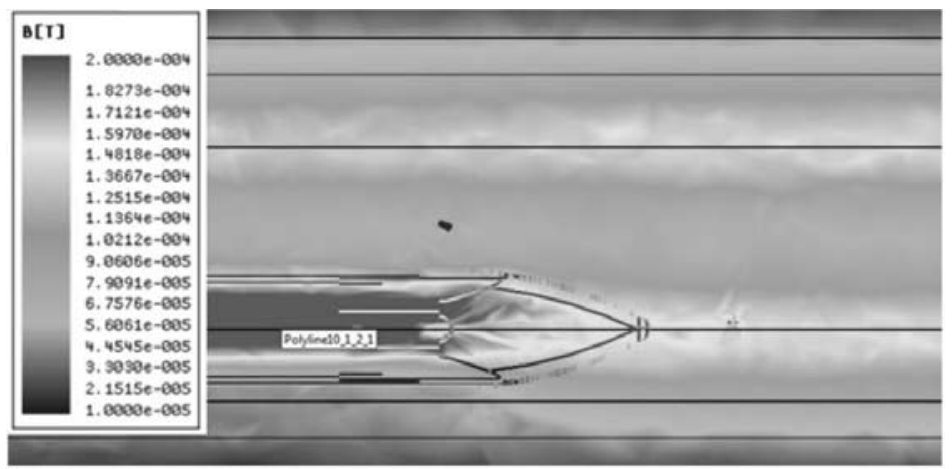

Fig. 6. Magnetic field distribution from top view of the model. The chosen plane is in the $x y$ plane $(z$ $=4)$, i.e., the plane right above the train. The outline of the train is denoted and the lines across the plot are the conductors including catenary wires, return conductors, and negative feeders.

Figures 7 and 8 are plots of the magnetic field variation along the rail in the $y$ direction. The plot starts from one end of the train and extends to $400 \mathrm{~m}$ to cover the entire model of the train and the railway system. From the magnetic field variation above the train shown in Fig. 7, the magnetic field in the region of the train $(0-196 \mathrm{~m})$ is approximately $80 \mu \mathrm{T}$ higher than that in the region without the train $(196-400 \mathrm{~m})$. From the magnetic field variation at the side of the train shown in Fig. 8, it can be seen that the magnetic field in the region of the train $(0-196 \mathrm{~m})$ is approximately $40 \mu \mathrm{T}$ lower than that in the region without the train $(196-400 \mathrm{~m})$. An interesting feature is that there are some pulses in the magnetic field variation. These pulses occur at the gaps between rolling stocks. At these gaps, there are no ferromagnetic materials, and thus, there are 


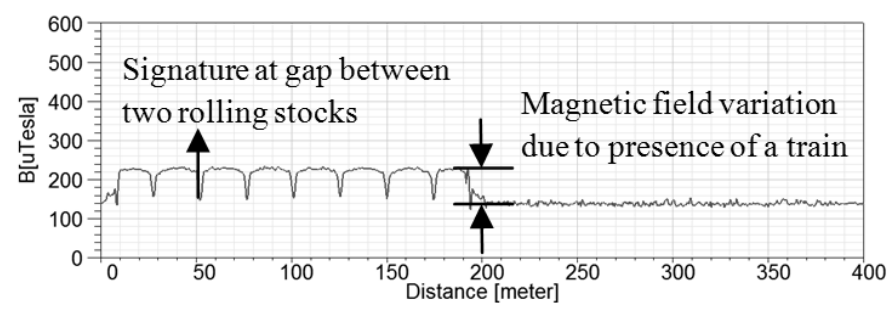

Fig. 7. Magnetic field variation along the $y$ direction $(x=0, z=4)$ above the train. The train ranges from 0 to $196 \mathrm{~m}$ in the plot. The signature change in magnetic field can be observed at the gaps between rolling stocks.

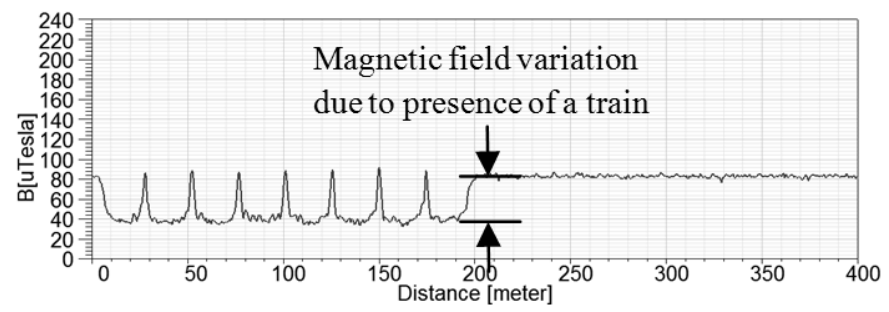

Fig. 8. Magnetic field intensity variation along the $y$ direction $(x=2, z=4)$ at the side of the train. The line lies in the $y$ direction and passes $(2,0,4)$. The train ranges from 0 to $196 \mathrm{~m}$ in the plot.

sudden changes in the magnetic field. This signature pattern can be used for counting the number of rolling stocks as explained in $\S 3.3 .2$.

From the above results, as a train passes, the magnetic field exhibits such patterns of variation and these can provide effective evidence for train presence detection. Since GMR sensors can sense a magnetic field from DC to over $1 \mathrm{MHz}$, stationary trains can also be detected.

\subsection{Best positions for sensors}

To determine where to place the magnetic field sensors, a few positions were analyzed for their suitability for placing sensors for train detection. The positions were chosen from above the train (A-C) and at the sides of the train (D-I) as shown in Fig. 9. Figures 10-12 illustrate the warping of the magnetic field due to the train along the $y$ direction from 0 to $400 \mathrm{~m}$ at these positions. From Fig. 10, it can be seen that the magnetic field variation differs at different heights above the train and it is larger for positions closer to the train. As it goes farther from the train in the $z$ direction, the variation becomes insignificant. From Fig. 11, it can be seen that at the side of the train, the magnetic field variation is larger for positions E and F. From Fig. 12, it can be seen that at the side of the train, the magnetic field variation is larger for positions closer to the train. When it is farther away from the train in the $x$ direction, the variation becomes smaller. 


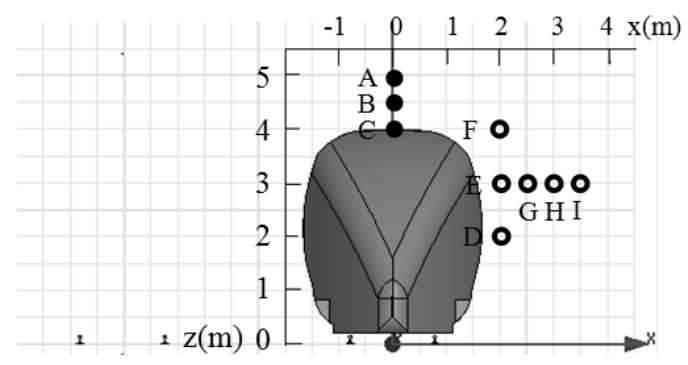

Fig. 9. Illustration of the positions selected for investigating the magnetic field variation. Dots are positions above the train and circles are positions at the side of the train. A-I correspond to the plots marked by A-I in Figs. 11-13. The positions A-C were chosen to illustrate the difference in the magnetic field variation at different vertical heights above the train. The positions D-F were chosen to illustrate the difference in the magnetic field variation at different vertical heights at the side of the train. The positions E and G-I were chosen to illustrate the difference in the magnetic field variation at different horizontal distances from the side of the train.
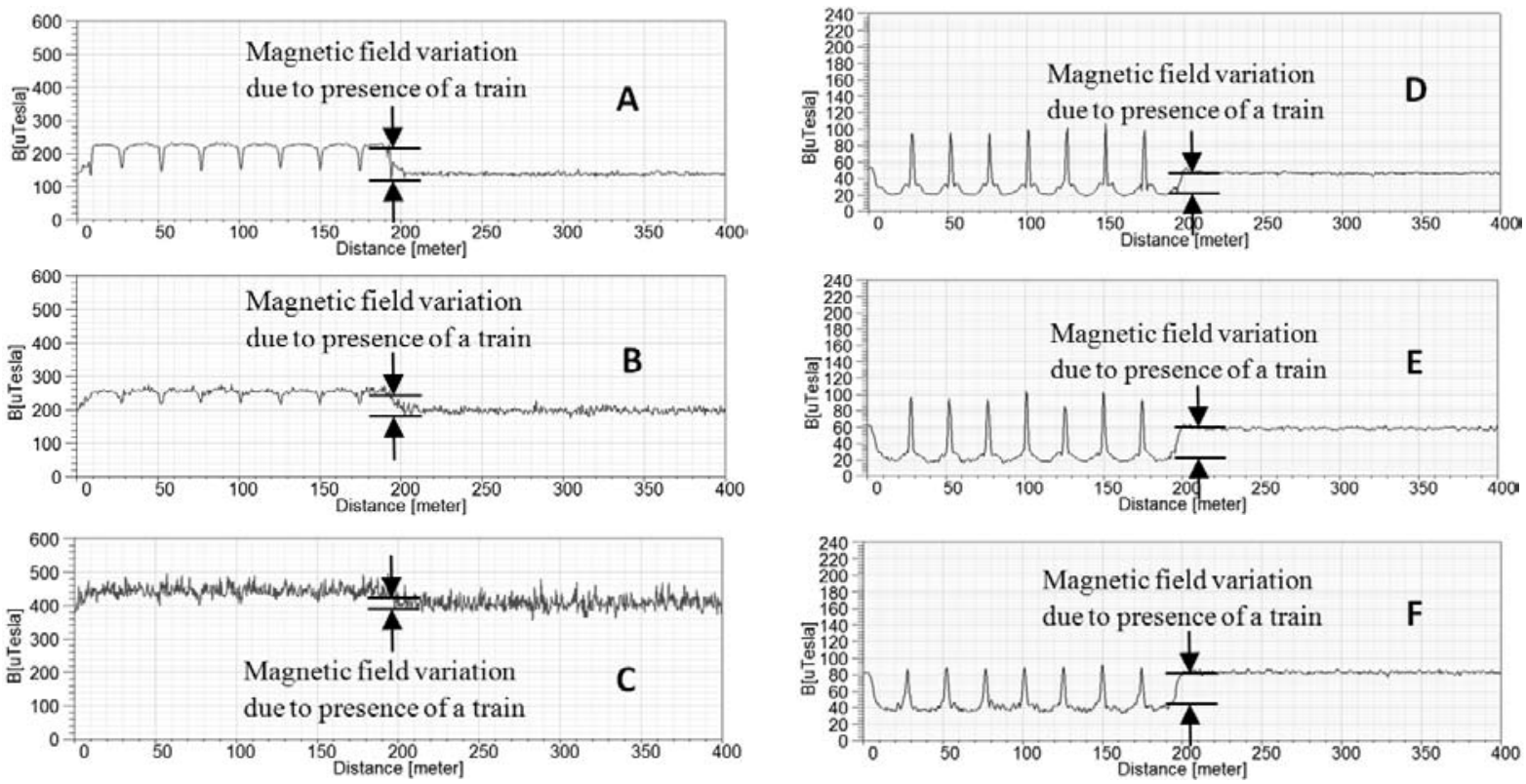

Fig. 10 (left). Magnetic field variation above the train at positions A-C: A, along the line passing (0, $0,4) ; \mathrm{B}$, along the line passing $(0,0,4.5)$; and $\mathrm{C}$, along the line passing $(0,0,5)$.

Fig. 11 (right). Magnetic field variation at the side of the train at positions D-F: D, along the line passing $(2,0,2)$; E, along the line passing $(2,0,3)$; and $\mathrm{F}$, along the line passing $(2,0,4)$. 

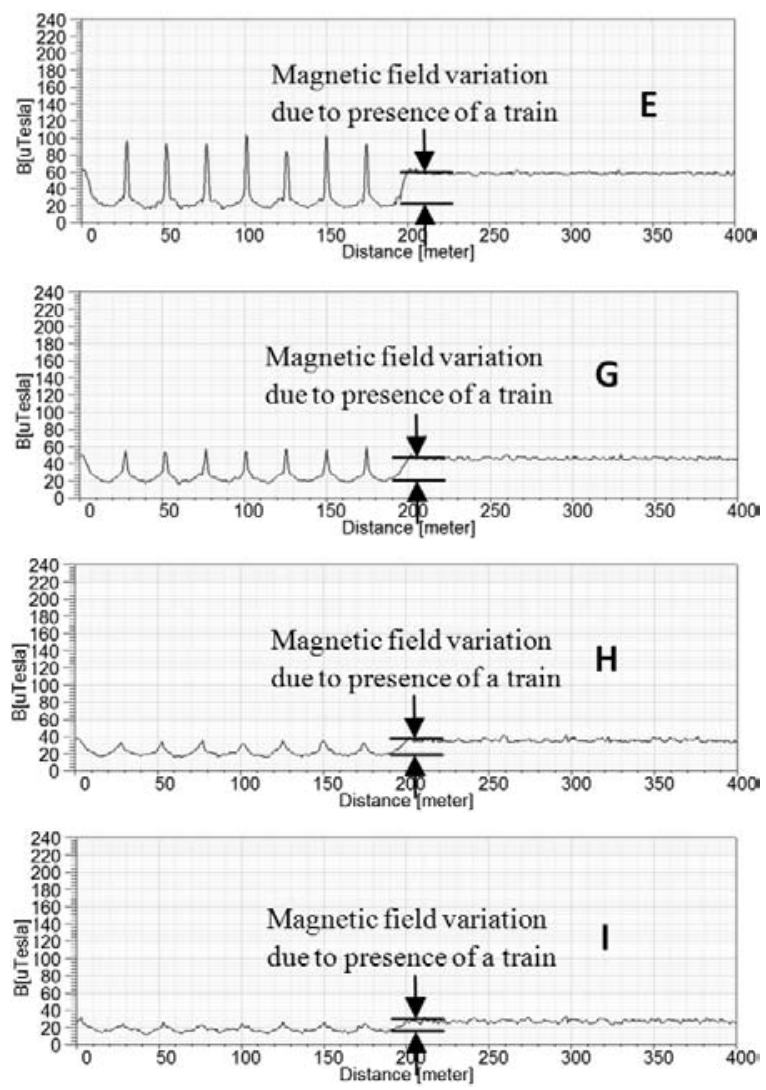

Fig. 12. Magnetic field variation at the side of the train at positions $\mathrm{E}$ and $\mathrm{G}-\mathrm{I}$ : $\mathrm{E}$, along the line passing $(2,0,3)$; G, along the line passing $(2.5,0,3)$; $\mathrm{H}$, along the line passing $(3,0,3)$; and $\mathrm{I}$, along the line passing $(3.5,0,3)$.

From the above observation and discussion, the warping of the magnetic field due to the train presence can be summarized as follows: above the train, the warping is stronger at positions closer to the train in the $z$ direction; at the side of the train, the warping is stronger at the vertical levels of $z=3$ and $z=4$ than at $z=2$, and it is stronger at positions with a shorter horizontal distance from the train. Therefore, the highlighted regions including positions $\mathrm{C}, \mathrm{E}$, and $\mathrm{F}$ in Fig. 13 are better for placing sensors because the warping of the magnetic field in these regions is stronger than in the other regions, and thus, a better signal-to-noise ratio is obtained. These regions can be accessed for placing sensors with a simple structure as shown in Fig. 14. On the basis of the findings described in $\S \S 3.1$ and 3.2, the magnetic field measurement can be applied to the detection of a set of parameters concerning the train: presence, number of rolling stocks, speed, and length. 


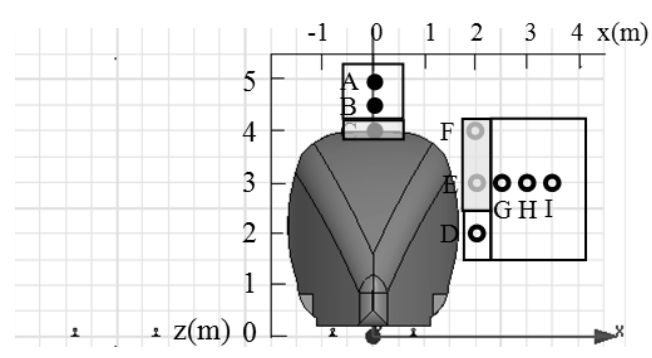

Fig. 13. Regions for placing magnetic sensors. The light-grey rectangular regions are better places for sensors with stronger warping effect of magnetic field from the train.

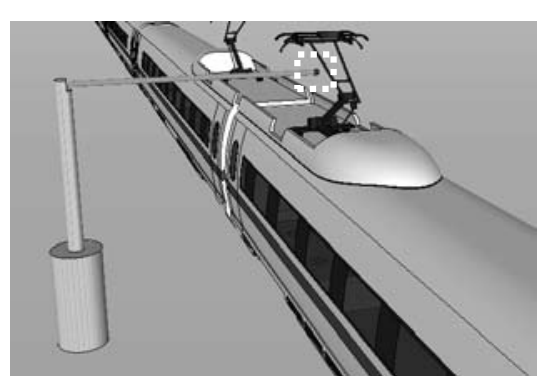

(a)

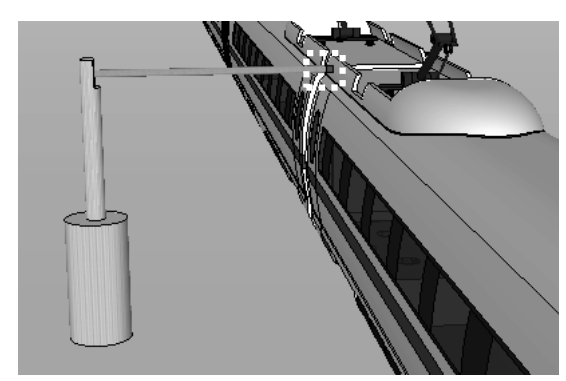

(b)

Fig. 14. Sensors placed (a) above the train and (b) at side of the train. The highlighted component enclosed by a dotted line at the end of the bar is the GMR sensor.

\subsection{Detection of trains}

\subsubsection{Detection of train presence}

As discussed in $\S 3.1$, the magnetic field above the train is higher than in the case without the train (Fig. 7), whereas the magnetic field is lower at the side of the train than in the case without the train (Fig. 8). Therefore, when a train passes by the sensor or stops next to the sensor, the magnetic field measured becomes higher (if the sensor is above the train) or lower (if the sensor is by the side of the train) than the normal level. Therefore, the presence of a train can be detected. When the train departs from the sensor, the magnetic field returns to the normal level again.

\subsubsection{Detection of number of rolling stocks}

In addition to the presence of a train, the magnetic field signatures indicating the gaps between rolling stocks (discussed in $\S 3.1$ ) can provide information for counting the number of rolling stocks. The number of rolling stocks is one plus the number of signatures detected by the sensor during the passing of the train. If two sensors are placed at the two ends of a track section, the number of rolling stocks that enter and 
leave this section can be recorded and compared to see if the whole train has left the track section.

\subsubsection{Detection of Speed}

There are two methods of speed detection. With one sensor and the known length of each rolling stock, the speed of the train can be deduced. As shown in Fig. 15, a train passes by sensor A, which is placed at position E denoted in Fig. 9. After the first rolling stock travels past sensor A, the gap between the rolling stocks and, thus, its signature is detected by sensor A at time $t_{1}$. After the second rolling stocks travel past sensor A, the second gap and, thus, its signature occur at time $t_{2}$. If the length of each rolling stocks is given as $L$ and the spacing between two rolling stocks is $d$, then the average speed $v$ of the train in this duration is

$$
v=\frac{L+2\left(\frac{d}{2}\right)}{t_{2}-t_{1}} .
$$

The train speed can also be detected with two sensors placed along the rail. In this case, the length of the rolling stock does not need to be predetermined. As shown in Fig. 16, a train passes by sensors B and C, which are both placed at position E denoted in Fig. 9. Suppose the occurrence of the first signature is taken as the sign for measuring the speed, sensor B detects its first signature at time $t_{3}$, and sensor $\mathrm{C}$ detects its first signature at time $t_{4}$. The distance $L_{\mathrm{BC}}$ between sensors $\mathrm{B}$ and $\mathrm{C}$ is known, and the approximate speed of the train can be obtained as

$$
v=\frac{L_{\mathrm{BC}}}{t_{4}-t_{3}} .
$$

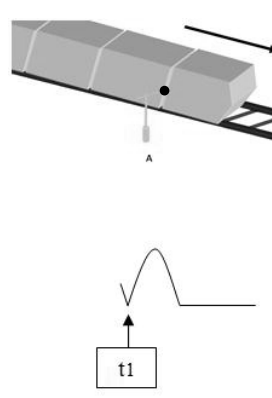

(a)
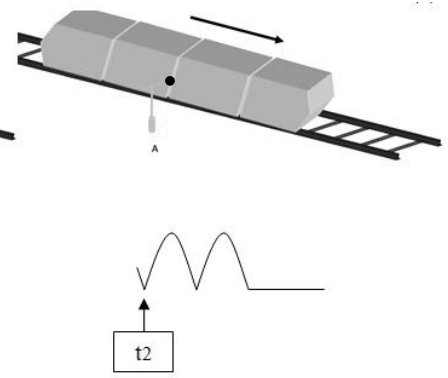

(b)

Fig. 15. Illustration for speed measurement with one sensor. A train is passing by sensor A from time $t_{1}$ to $t_{2}$. The situations at (a) $t_{1}$ and (b) $t_{2}$ are shown. The components marked by $\bullet$ are the GMR sensors. The arrow indicates the traveling direction of the train. The waveforms below are the corresponding magnetic field variation. It can be seen that the first signature happens at $t_{1}$ and the second one occurs at $t_{2}$. 


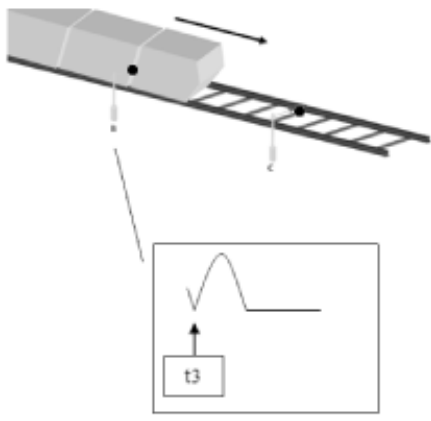

(a)

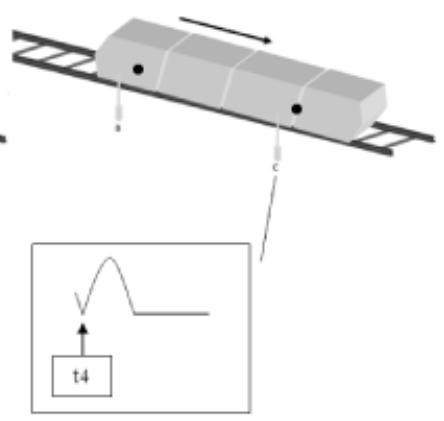

(b)

Fig.16. Illustration for speed measurement with two sensors. A train is passing sensor B and sensor $\mathrm{C}$ from time $t_{3}$ to $t_{4}$. The situations at (a) $t_{3}$ and (b) $t_{4}$ are shown. The components marked by $\bullet$ are the GMR sensors. The arrow indicates the traveling direction of the train. The waveforms below are the corresponding magnetic field variation. It can be seen that at $t_{3}$, sensor $\mathrm{B}$ has its first signature, while at $t_{4}$, sensor $\mathrm{C}$ has its first signature.

\subsubsection{Detection of length}

If the train speed is known, the train length can be derived by measuring the duration for the entire train to travel past the sensor. As shown in Fig. 17, a train passes by sensor D, which is placed at position $\mathrm{E}$. The head of the train passes the sensor at time $t_{5}$, whereas the tail passes the sensor at time $t_{6}$. Sensor $\mathrm{D}$ will detect the magnetic field variation owing to the train presence starting from time $t_{5}$ until $t_{6}$ when the train has left. If the speed of the train is $v$, the length of the train $l$ will be

$$
l=v\left(t_{6}-t_{5}\right)
$$

\subsection{Fail-safe mechanism}

Fail-safe is a necessary part of a complete train detection system. By defining proper logic levels for the magnetic field, our technique can be fail-safe. This can be realized when the sensor is placed at the side of the train, rather than above the train. Taking the magnetic field variation at position $\mathrm{E}$ as an illustration, the red line at level $40 \mu \mathrm{T}$ is taken as the reference line (Fig. 18). The comparator defines the magnetic field above this reference line as logic " 1 ", and that below as logic " 0 ". When there is no train and the sensor works well, the magnetic field measured is above the reference line and thus the comparator outputs " 1 ", which indicates a proceed signal. In the presence of a train, the magnetic field drops to the lower level and the comparator outputs " 0 ", which indicates a stop signal. If the sensor fails, there is no magnetic field detected and the comparator will keep providing a logical output of 0 indicating a stop signal. We define variable $a$ as the presence status of a train ("0" = no train, " $1 "=$ train presence), variable $b$ as the working status of the sensor (" 0 " = fail, " 1 " = working), and $s$ as the logical output of the comparator (" 0 " = occupied, " $1 "=$ unoccupied). The relation of $a, b$, and $s$ is described using eq. (4) and the logic table is shown in Table 4.

$$
s=\bar{a} * b
$$




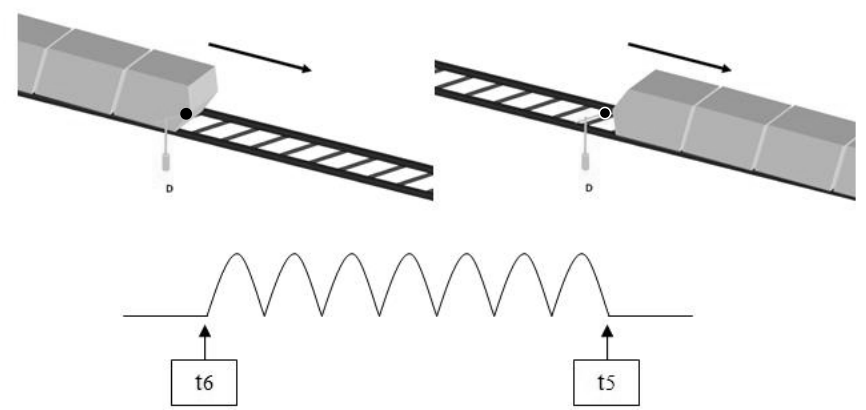

(a)

(b)

Fig. 17. Illustration of train length measurement with one sensor. A train is passing sensor D from time $t_{5}$ to $t_{6}$. The situations at (a) $t_{5}$ and (b) $t_{6}$ are shown. The arrow indicates the traveling direction of the train. The waveforms below are the corresponding magnetic field variation. It can be seen that the variation starts at $t_{5}$ and ends at $t_{6}$. This means that the entire train passes from $t_{5}$ to $t_{6}$.

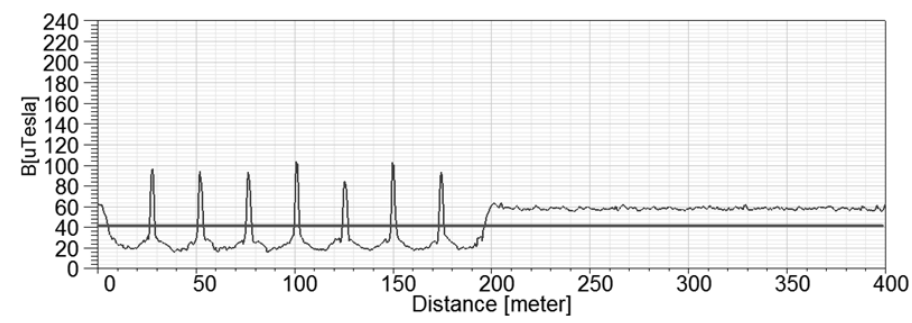

Fig. 18. Mapping of analog value of magnetic field intensity to logics "1" and " 0 ". The red line at $40 \mu \mathrm{T}$ is chosen to be the reference line. The value above the reference line is logic " 1 ", below is

Table 4

Logic table for different cases of train presence and sensor working status. "a", "b", and "s" are the presence status of a train, the working status of the sensor, and the logic output of the comparator, respectively.

\begin{tabular}{lcccc}
\hline Cases & $\mathrm{a}$ & $\mathrm{b}$ & $\mathrm{s}$ & Signal \\
\hline No train \& device works & 0 & 1 & 1 & Proceed \\
With train \& device works & 1 & 1 & 0 & Stop \\
No train \& device fails & 0 & 0 & 0 & Stop \\
With train \& device fails & 1 & 0 & 0 & Stop \\
\hline
\end{tabular}

It can be seen that the detection system produces a logic "1", i.e., a "proceed" aspect signal, only in the case when there is no train and the device works normally. The signal is "stop" for all other cases including the cases when the sensor fails. This meets the fail-safe principle. 


\section{Conclusions}

The new technique for train detection overcomes some of the shortcomings of track circuits. Firstly, it does not require special features of tracks, such as insulated rail joints; secondly, it does not lose effectiveness at grade crossing. Moreover, the detection is immune to factors such as rust and wet environment. The new technique also has advantages in terms of cost, size, and energy consumption. GMR sensors cost just a few US dollars each. A GMR sensor is only a few $\mathrm{mm}$ in size, which leaves plenty of margin for construction space. Its power consumption is only around $10 \mathrm{~mW}^{(21)}$ and, thus, a battery can last for a long time without maintenance. The new train detection system has a relatively low complexity because it does not require transmitter devices or wayside controllers. Unlike some of the train detection methods that require additional processing of the raw detection data, for example, the image video detection technique, the GMR sensor converts the magnetic field measurement into electrical signal directly. This technique is proposed and described in detail, and its principle and feasibility are verified by modeling and simulation in this study. Its advantages over the existing methods make it promising for train detection in modern high-speed railway systems.

\section{References}

1 W. M. Hwang and Y. L. Huang: Trans. Can. Soc. Mech. Eng. 29 (2005) 41.

2 A. K. W. Ahmed, C. Liu and I. Haque: Trans. Can. Soc. Mech. Eng. 20 (1996) 365.

3 Y. Ravalard, D. Coutellier and S. Masfrand: Trans. Can. Soc. Mech. Eng. 18 (1994) 269.

4 M. A. Soliman, M. S. Kaldas, D. C. Barton and P. C. Brooks: IJETI 2 (2012) 85.

5 J. I. Real, L. Montalban, T. Real and V. Puig: J. Vibroeng. 14 (2012) 813.

6 D. Chen, W. Xie and C. Qian: Foreign Electron. Meas. Technol. 24 (2005) 9.

7 J. Gonzalez, C. Rodriguez, J. Blanquer, J. M. Mera, E. Castellote and R. Santos: J. Adv. Transp. 44 (2010) 53.

8 J. W. Palmer: RSCS 2012 (2012) 60.

9 N. Nikolov and N. Nedelchev: IEEE Proc. Electr. Power Appl. 152 (2005) 1049.

10 A. Taguti: Electr. Eng. Jpn. 127 (1999) 64.

11 F. T. Pascoe: United States Patent No. 3725699 (1973).

12 X. Wu: RSCE 3 (2006) 43.

13 H. Uebel: United States Patent No. 6565046 (2003).

14 L. L. McAllister: European Patent Specification No. 1295775 (2011).

15 A. A. Carroll, J. E. Gordon, R. P. Reiff and S. E. Gage: Proc. Seventh International Symposium on Railroad-Highway Grade Crossing Research and Safety: Getting Active at Passive Crossings (ARRB Group Limited, Melbourne, 2002) pp. 36-49.

16 D. Guzas: J. Vibroeng. 12 (2010) 642.

17 J. I. Real, T. Asensio, L. Montalban and C. Zamorano: J. Vibroeng. 14 (2012) 880.

18 J. I. Real, T. Asensio, L. Montalban and C. Zamorano: J. Vibroeng. 14 (2012) 408.

19 J. Pelegri, J. Alberola and V. Llario: Proc. 28th IECON (IEEE, Sevilla, 2002) pp. 1693-1695.

20 NVE Corp: Low-Field Magnetic Sensing with GMR Sensors, http://www.nve.com/ Downloads/lowfield.pdf (accessed on January 2013).

21 NVE Corp: AA and AB-Series Analog Sensors, http://www.nve.com/Downloads/ analog_catalog.pdf (accessed on May 2012). 